\title{
Identificação da carga de trabalho da enfermagem em Centro de Material e Esterilização*
}

\author{
Identification of nursing workload in the Sterile Processing Department \\ Identificación de la carga de trabajo de enfermería en el Centro de Material y Esterilización
}

Como citar este artigo:

Costa JA, Fugulin FMT. Identification of nursing workload in the Sterile Processing Department. Rev Esc Enferm USP. 2020;54:e03621. doi: https://doi. org/10.1590/S1980-220X2019004203621

\section{Janaína Anchieta Costa ${ }^{1}$}

Fernanda Maria Togeiro Fugulin²

* Extraído da tese: "Método para dimensionamento de pessoal de enfermagem em Centro de Material e Esterilização (CME)", Programa de Pós-Graduação em Gerenciamento em Enfermagem, Escola de Enfermagem da Universidade de São Paulo, 2015.

${ }^{1}$ Universidade de São Paulo, Escola de Enfermagem, Programa de PósGraduação em Gerenciamento em Enfermagem, São Paulo, SP, Brasil.

${ }^{2}$ Universidade de São Paulo, Escola de Enfermagem, Departamento de Orientação Profissional, São Paulo, SP, Brasil.

\begin{abstract}
Objective: To determine the workload in the Sterile Processing Department as a parameter for the method of nursing staff sizing. Method: Quantitative, observational study conducted in four hospitals in the city of São Paulo, in three steps: mapping of activities in work positions; determination of the standard processing time of materials; and establishment of the average daily workload. Results: In the 15 mapped work positions, 1315 samples of time were obtained. As the time values did not show significant differences, it was possible to determine the standard processing time of materials. The average daily workloads corresponded to $217.4 ; 294.0 ; 123.1$; and 189.1 hours. Conclusion: In addition to proposing a method to determine the workload, the present study indicates parameters for the processing of materials and serves as an important reference to develop consistent proposals for the planning, allocation and distribution of nursing staff in these departments.
\end{abstract}

\section{DESCRIPTORS}

Nursing Staff; Workload; Sterilization; Materials Management; Hospital. 


\section{INTRODUÇÃO}

Um dos recursos mais importantes, com papel fundamental e impacto direto na qualidade dos serviços de saúde oferecidos à população são os recursos humanos. Quando sua gestão é realizada de forma apropriada, produz resultados que contribuem, sobremaneira, para o desenvolvimento do trabalho e o alcance dos objetivos do setor.

Para acompanhar a evolução nas empresas modernas em constantes e profundas mudanças em todo o mundo, o desempenho dos serviços de saúde, de forma geral, dependerá de investimentos nas pessoas, da oferta de condições de trabalho satisfatórias e do estabelecimento de uma cultura organizacional democrática e incentivadora, que promova e sustente melhorias contínuas nos processos de trabalho ${ }^{(1)}$.

Nessa perspectiva, as instituições de saúde devem buscar estratégias de gestão capazes de conciliar o atendimento das necessidades de saúde dos usuários e a obtenção de resultados econômicos satisfatórios para sua permanência no mercado, de forma a equilibrar os custos operacionais e otimizar os recursos disponíveis e necessários para manter a qualidade e segurança nos serviços oferecidos à população.

O desconhecimento de ferramentas de controle dos gastos e redução de despesas tem repercutido diretamente na política de recursos humanos das instituições de saúde. Além disso, afeta principalmente o quadro de profissionais da equipe de enfermagem, que representam o maior quantitativo de pessoal e, consequentemente, o maior componente dos orçamentos operacionais dessas organizações ${ }^{(2-3)}$.

Apesar do papel relevante que desempenham na qualidade do processo assistencial, as unidades de Centro de Material e Esterilização (CME), particularmente aquelas inseridas em instituições hospitalares, são as mais afetadas. Talvez pela ausência de contato com as atividades de cuidados assistenciais junto aos pacientes e seus familiares, ainda contam com um quadro de pessoal insuficiente ou sem qualificação adequada para desenvolver as atividades de processamento dos produtos para a saúde ${ }^{(2)}$.

A insuficiência de métodos de dimensionamento de pessoal que considerassem a especificidade do processo de trabalho desenvolvido na unidade contribuiu para a permanência desse cenário nas unidades de $\mathrm{CME}$, o que prejudicou a realização de estimativas e avaliações do quadro de pessoal, ao mesmo tempo em que forneceu elementos de auxílio aos enfermeiros na argumentação e justificativa das propostas relacionadas à contratação de pessoal adicional junto aos administradores das instituições hospitalares ${ }^{(2)}$.

A principal variável dos métodos de dimensionamento de pessoal de enfermagem, consolidados para as unidades assistenciais, diz respeito à determinação da carga de trabalho, ou seja, o tempo médio diário de trabalho necessário para atender as demandas assistenciais dos pacientes. Nessa perspectiva, a carga de trabalho em CMEs pode ser obtida a partir da determinação do tempo médio despendido na execução de cada atividade envolvida nas diferentes etapas do processamento de materiais, favorecendo a operacionalização do planejamento e a avaliação do quantitativo de pessoal de enfermagem.

Após explicitar as atividades realizadas nas unidades de CME, esse referencial pode ser utilizado para identificar o tempo despendido no desenvolvimento dessas ações, como subsídio para determinar a carga de trabalho de enfermagem e propor um método de dimensionamento específico para essas unidades.

Em 2017, o Conselho Federal de Enfermagem (COFEN), por meio da Resolução n ${ }^{\circ}$ 543, atualizou e estabeleceu os parâmetros mínimos para dimensionar o quantitativo de profissionais das diferentes categorias de enfermagem para os serviços e locais onde são realizadas atividades de enfermagem ${ }^{(4)}$.

Para definir a carga de trabalho dos profissionais de enfermagem nas unidades de CME, essa Resolução considera a produção da unidade, multiplicada pelo tempo padrão das atividades realizadas nas diferentes áreas de trabalho, conforme o referencial adotado nesse estudo ${ }^{(4)}$.

Assim, a presente pesquisa tem o objetivo de determinar a carga de trabalho em CMEs como parâmetro mínimo para o método de dimensionamento de profissionais de enfermagem. Dessa forma, contribuirá para superar as dificuldades que envolvem aspectos quantitativos e qualitativos de profissionais nas unidades de CME e para o avanço do conhecimento na área do gerenciamento de recursos humanos em enfermagem.

\section{MÉTODO}

\section{TIPO DO ESTUDO}

Trata-se de um estudo de natureza quantitativa, observacional, do tipo desenvolvimento metodológico ${ }^{(5)}$.

\section{Cenário}

A pesquisa foi realizada em quatro unidades de CME de instituições hospitalares gerais públicas e privadas de grande porte da cidade de São Paulo.

\section{Critérios de Seleção}

Para participar desse estudo, foram selecionadas unidades de CME que atendessem aos critérios mínimos de boas práticas para o processamento de produtos para saúde, formulados a partir dos regulamentos e manuais técnicos da área ${ }^{(6-13)}$ : centralização das atividades pertinentes ao processamento dos produtos para saúde; utilização de equipamentos nas diferentes etapas do processamento dos produtos para saúde; realização de controles de produção nas diferentes áreas da unidade; presença de enfermeiros, além daquele responsável pela unidade.

O número de unidades foi estabelecido considerando a probabilidade de obter valores de tempo semelhantes ou divergentes entre as instituições. Inicialmente, os dados seriam coletados em três CMEs. Caso apresentassem diferença significativa, ao nível de $95 \%$, seriam verificados em uma quarta instituição.

As unidades de CME que aceitaram participar da pesquisa foram denominadas CME I, CME II, CME III e CME IV. 
O CME I e o CME II pertenciam a hospitais privados, que na época da coleta dos dados contavam, respectivamente, com 318 e 313 leitos de internação e 14 e 10 salas cirúrgicas.

O CME III e o CME IV localizavam-se em instituições de saúde públicas, que na época da coleta dos dados possuíam, respectivamente, 236 e 499 leitos de internação e 14 e 22 salas operatórias.

\section{Coleta de Dados}

Esta pesquisa foi conduzida em três etapas metodológicas:

\section{ETAPA 1: MAPEAMENTO DAS ATIVIDADES REALIZADAS EM CME EM OPERAÇÕES DE PRODUÇÃO OU POSIÇÕES DE TRABALHO}

As atividades realizadas pela enfermagem em unidades de CME foram identificadas na literatura, em estudo que elaborou um quadro de atividades validado por enfermeiras e representativo das ações desenvolvidas no CME. Esse quadro possui seis áreas de trabalho, definidas e apresentadas como processos de trabalho específicos, 25 subprocessos e 110 atividades, além de 25 atividades específicas das enfermeiras ${ }^{(2)}$.

As atividades identificadas no referido estudo ${ }^{(2)}$ foram reorganizadas e agrupadas em operações de produção, que neste estudo, foram designadas como posições de trabalho, segundo o fluxo das ações desenvolvidas pelos profissionais dentro de sua jornada.

Para determinar as posições de trabalho, foram observados e descritos os fluxos de desenvolvimento das atividades que integram o processamento de produtos para saúde nas diferentes áreas de trabalho do CME.

Inicialmente, o fluxo das atividades foi observado na primeira instituição que aceitou participar da pesquisa. $\mathrm{O}$ produto foi um instrumento constituído por 15 posições de trabalho, que representou integralmente os processos e fluxos de trabalho executados nas unidades estudadas.

Cada uma das áreas de trabalho do CME representou um processo de trabalho específico, composto por diferentes operações de produção visualizadas em posições de trabalho.

Para verificar a viabilidade de aplicação do instrumento nas demais unidades participando do estudo, ele foi apresentado e discutido com as enfermeiras das outras instituições antes da coleta dos dados, para evitar dificuldades que comprometessem sua aplicação. As enfermeiras consultadas consideraram o instrumento pertinente e representativo das posições de trabalho das suas unidades.

\section{ETAPA 2: DETERMINAÇÃO DO TEMPO PADRÃO DE PROCESSAMENTO DOS MATERIAIS EM CADA POSIÇÃO DE TRABALHO}

Para estabelecer o tempo padrão de processamento dos materiais em cada posição de trabalho, foi identificado, por meio da cronometragem, o tempo médio gasto pelos trabalhadores para desenvolver as atividades que compõem a posição de trabalho.

$\mathrm{Na}$ cronometragem, a leitura do tempo é contínua e direta do início ao fim da atividade, com o auxílio de um cronômetro. O método para uso do cronômetro foi definido a partir da Leitura Repetitiva, que consiste em acionar o aparelho a cada início de mensuração, registrar a leitura e zerar o aparelho, iniciando uma nova medida ${ }^{(14)}$.

A cronometragem do tempo despendido pelos profissionais foi realizada por observadoras de campo com formação na área e domínio do processo de trabalho desenvolvido na unidade. Elas foram orientadas quanto ao objetivo da pesquisa, ao instrumento contendo as posições de trabalho identificadas e as respectivas atividades desenvolvidas em cada uma delas, além de todo o procedimento envolvendo a coleta de dados.

Para verificar a aplicabilidade do instrumento construído, identificar possíveis dificuldades com sua utilização nas unidades de CME, e averiguar o grau de confiabilidade na mensuração do tempo das posições de trabalho, foi realizado um teste de concordância entre a pesquisadora (considerada referência) e as observadoras.

No primeiro dia de coleta de dados, a pesquisadora acompanhou a mensuração da primeira amostra de tempo em todas as posições de trabalho realizadas por cada observadora. Nos demais dias de coleta, a pesquisadora ficou à disposição das observadoras para esclarecer dúvidas e acompanhou o encerramento da coleta, a partir da verificação do número de amostras encontradas.

Dada a dificuldade de medir o tempo de processamento dos produtos nas diferentes posições de trabalho, devido à sua heterogeneidade, na investigação do tempo, foram considerados os itens envolvidos em cada ciclo de trabalho observado $(N)$ nas diferentes posições de trabalho.

Cada ciclo de trabalho $(N)$ foi cronometrado e o resultado do seu tempo de execução $\left(T_{S}\right)$ foi anotado pela observadora de campo na planilha de coleta dos dados. Como a planilha foi construída de forma informatizada, ao registrar o tempo do ciclo de trabalho $\left(T_{S L}\right)$, o tempo médio $\overline{(T E)_{S_{i}}}$, o desvio padrão $(s)$, o coeficiente de variação $(c v)$ e o tamanho da amostra $\left(N^{\prime}\right)$ eram calculados automaticamente.

Por exemplo, o tempo médio $\overline{(T E)_{S_{i}}}$ das atividades que compõem o processo de trabalho $(S)$ da posição de trabalho 1 foi calculado a partir da seguinte equação:

$$
\overline{(T E)_{S_{i}}}=\frac{1}{N_{S_{l}}} \sum_{l=1}^{N} T_{S_{l}}
$$

Na equação proposta, $N_{S l}$ é a quantidade total de ciclos de trabalho amostrados (tamanho da amostra) e $T_{S l}$ é tempo de execução dos ciclos de trabalho amostrados, cronometrados pelas observadoras de campo em cada CME das instituições que participaram da pesquisa.

Além da investigação do tempo médio das atividades, a produção diária de cada posição de trabalho foi analisada para determinar o tempo médio de processamento dos produtos para saúde nas diferentes posições de trabalho do CME.

Como os processos de trabalho desenvolvidos em cada área do CME são específicos, a produção foi estabelecida de forma distinta. No Quadro 1, são apresentados os indicadores de produção considerados em cada uma das áreas de trabalho do CME. 
Quadro 1 - Indicadores de produção de cada posição de trabalho das unidades de CME - São Paulo, SP, Brasil, 2014.

\begin{tabular}{|c|c|c|}
\hline Área & Posição de trabalho & Indicador de produção \\
\hline \multirow{2}{*}{ A - Suja ou contaminada (expurgo) } & 1) Recepção de materiais das unidades consumidoras & Quantidade de kits recebidos pelo CME \\
\hline & 2) Limpeza dos materiais & Quantidade de kits processados pelo CME \\
\hline \multirow{3}{*}{ B - Controle de materiais em consignação } & 3) Recepção de materiais em consignação & Quantidade de kits recebidos \\
\hline & 4) Conferência dos materiais em consignação após a cirurgia & Quantidade de kits conferidos \\
\hline & $\begin{array}{l}\text { 5) Devolução dos materiais em consignação junto às } \\
\text { empresas fornecedoras }\end{array}$ & Quantidade de kits devolvidos \\
\hline \multirow{4}{*}{ C - Preparo de materiais } & $\begin{array}{l}\text { 6) Secagem dos materiais e distribuição nas bancadas } \\
\text { de preparo }\end{array}$ & Quantidade de kits processados pelo CME \\
\hline & $\begin{array}{l}\text { 7) Inspeção, teste e separação dos materiais ou } \\
\text { instrumentais limpos }\end{array}$ & $\begin{array}{l}\text { Quantidade de kits de instrumental } \\
\text { processados }\end{array}$ \\
\hline & 8) Montagem e embalagem dos materiais e instrumentais & $\begin{array}{l}\text { Quantidade de kits de instrumental } \\
\text { processados }\end{array}$ \\
\hline & $\begin{array}{l}\text { 9) Montagem e embalagem dos kits ou circuitos de } \\
\text { assistência ventilatória }\end{array}$ & $\begin{array}{l}\text { Quantidade de kits de assistência } \\
\text { ventilatória processados }\end{array}$ \\
\hline D - Esterilização de Materiais & $\begin{array}{l}\text { 10) Montagem da carga e acompanhamento do ciclo de } \\
\text { esterilização }\end{array}$ & Quantidade de cargas/ciclos realizados \\
\hline \multirow{5}{*}{$\begin{array}{l}\text { E - Armazenamento e distribuição de } \\
\text { materiais estéreis }\end{array}$} & $\begin{array}{l}\text { 11) Retirada da carga estéril dos equipamentos e } \\
\text { verificação da efetividade do processo de esterilização }\end{array}$ & Quantidade de cargas/ciclos realizados \\
\hline & 12) Guarda dos materiais e roupas estéreis & Quantidade de cargas/ciclos realizados \\
\hline & $\begin{array}{l}\text { 13) Montagem dos carros de transporte às unidades } \\
\text { consumidoras }\end{array}$ & Quantidade de carros montados \\
\hline & $\begin{array}{l}\text { 14) Organização e controle do ambiente e dos materiais } \\
\text { estéreis }\end{array}$ & Quantidade de kits processados pelo CME \\
\hline & $\begin{array}{l}\text { 15) Distribuição dos materiais e roupas estéreis às } \\
\text { unidades consumidoras }\end{array}$ & Quantidade de kits processados pelo CME \\
\hline
\end{tabular}

$\mathrm{Na}$ presente pesquisa, os kits foram considerados como um conjunto de elementos, peças ou materiais montados com uma finalidade específica.

Para transformar os itens envolvidos no desenvolvimento de cada ciclo de trabalho mensurado em kits produzidos, o tempo médio de processamento de todos os ciclos obtidos em uma dada posição de trabalho foi comparado com o tempo de cada ciclo individualmente. Assim foi possível determinar, por equivalência, o número provável de kits produzidos em cada intervalo de tempo cronometrado.

A equação a seguir representa os cálculos realizados para obter os tempos médios de execução para os kits:

$$
(\overline{T K})_{S_{i}}=\frac{\overline{(T E)_{S_{i}}}}{\bar{K}}
$$

O valor médio do tempo de execução para o kit $\overline{(T E)_{S_{i}}}$ em determinada posição de trabalho, foi calculado pela razão entre o valor médio do tempo obtido na execução do processamento dos itens $\overline{(T E)_{S_{i}}}$ envolvidos nos ciclos de trabalho mensurados, e o valor correspondente à quantidade média de kits $(\overline{\boldsymbol{K}})$, determinado pela equivalência.

Para determinar o tempo médio de execução dos kits produzidos em cada uma das posições de trabalho, foi calculada a média dos tempos de execução dos kits obtidos nas quatro unidades de CME. Foi considerado o nível de confiança $1-\alpha=0,95$ nos dados coletados e um erro relativo $\varepsilon \%=5 \%$ entre a média populacional e a média das amostras.

O tempo padrão de processamento do Kit $(T P)_{i}$ foi determinado pelo acréscimo do fator correspondente ao ritmo do trabalhador $(F R=1,0)$ na execução das atividades da sua posição de trabalho, e das tolerâncias correspondentes às necessidades pessoais e de fadiga do trabalhador $(\mathrm{FT}=1,09)$ ao tempo médio de execução dos kits $(T K)_{S_{i}}^{(14)}$.

$$
(T P)_{i}=(\overline{T K})_{S_{i}} \times F R \times F T
$$

O tamanho da amostra ( $N$ ') necessário para definir estatisticamente o tempo médio despendido em cada ciclo de trabalho foi obtido a partir das $\left(N^{\prime}\right)=$ cinco primeiras amos tragens. $\mathrm{O}$ número de observações $(N)$ para cada ciclo de trabalho foi calculado pela equação a seguir, adaptada para pequenas amostras ${ }^{(14)}$ :

$$
N^{\prime}=\left[\left(\frac{t_{95 \%}}{\epsilon}\right)\left(\frac{s}{\bar{X}}\right)\right]^{2}
$$

Onde:

$\left(\frac{s}{\bar{X}}\right)$ é o coeficiente de variação dos dados coletados;

$t_{95 \%}$ é o valor da distribuição t de Student para (N-1) graus de liberdade (GL);

$\varepsilon$ é o valor do erro relativo admitido entre a média da população e da amostra $(\varepsilon=0,05)$;

Considerando semelhantes tempos médios de execução para os kits obtidos nas diferentes posições de trabalho, inicialmente, a coleta de dados foi programada para realização no $\mathrm{CME}$ de três instituições hospitalares diferentes. Entretanto, como os tempos médios de várias amostras apresentaram diferença significativa ao nível de 95\% entre as instituições, a amostra foi ampliada para quatro instituições. 
ETAPA 3: EsTABELECIMENTO DA CARGA MÉDIA DIÁRIA DE TRABALHO EM CADA POSIÇÃO DE TRABALHO DO CME

A carga média diária de trabalho $\bar{T}_{i}$ da posição de trabalho 1 foi calculada pelo produto entre a quantidade média diária de produção $\overline{\Re_{i}}$ desta posição de trabalho e o tempo padrão $(T P)_{i}$ de processamento de cada kit.

$$
\overline{\left(T_{i}\right)}=(T P)_{i} \times \overline{\Re_{i}}
$$

A produção média diária $\overline{\Re_{i}}$ (quantidade de kits/dia) em cada posição de trabalho foi obtida junto às enfermeiras das instituições participantes do estudo, com base nos registros de produção existentes nas unidades.

A carga de trabalho média diária total do $\mathrm{CME} \overline{\mathrm{T}}_{\text {cme }}$ foi obtida pela soma das cargas de trabalho média de todas as posições de trabalho, conforme a seguinte equação:

$$
\overline{\mathrm{T}_{c m e}}=\sum_{l=1}^{15} \overline{\mathrm{T}}_{i}
$$

A partir da determinação da carga de trabalho, a quantidade de trabalhadores (técnicos e auxiliares de enfermagem) $q_{\text {tec }}$ necessária para executar a carga de trabalho do CME durante um dia de trabalho foi obtida pela equação:

$$
q_{\text {tec }}=\frac{\overline{\mathrm{T}_{c m e}}}{t}
$$

Onde:

t é o turno de trabalho, ou seja, o tempo da jornada de trabalho dos profissionais, que nas quatro instituições tem duração de seis horas.

$\overline{\mathrm{T}}_{\text {cme }}$ é a carga de trabalho média total do CME

A quantidade de trabalhadores necessária ao funcionamento do CME pode ser determinada pela quantidade diária de profissionais, acrescida do índice de segurança técnica $(\mathrm{IST}=15 \%)$ para cobertura de férias e ausências não planejadas, conforme Resolução COFEN n 543 de 2017(4).

$$
\mathrm{Q}_{c m e}=q_{t e c} \times(1+I S T)
$$

\section{ANÁlise E TRATAMENTO DOS DADOS}

Os dados coletados foram lançados em planilhas eletrônicas que compuseram um banco de dados específico para cada CME, facilitando a organização, consulta, análise e realização dos cálculos propostos.

Para as variáveis quantitativas, foram calculadas medidas de tendência central e de dispersão.

$\mathrm{O}$ quadro de profissionais esperado em cada CME, obtido pela aplicação do método proposto, foi comparado com o quadro existente nas diferentes Unidades utilizando-se o teste QUI quadrado. O nível de significância estabelecido para o teste foi de $5 \%$.

\section{AsPeCtos ÉTICOS}

A pesquisa foi aprovada pelo Comitê de Ética em Pesquisa da Escola de Enfermagem da Universidade de São Paulo, instituição proponente (Parecer no 623.136 em 22/04/2014) e posteriormente, pelas instituições que aceitaram participar do estudo. As diretrizes estabelecidas pela Resolução 466/12 do Conselho Nacional de Saúde foram obedecidas durante toda a coleta dos dados.

Os profissionais de enfermagem foram orientados sobre os objetivos da pesquisa, o caráter voluntário de participação, a garantia do anonimato e o direito de recusar-se a participar do estudo em qualquer momento, sem que isso acarretasse prejuízo de qualquer natureza. Os Termos de Consentimento Livre e Esclarecido foram assinados pelos profissionais antes do início da coleta de dados.

\section{RESULTADOS}

Para alcançar o número de amostras necessárias para estabelecer o tempo padrão nas posições de trabalho, foram observados 55 (100\%) profissionais de enfermagem, que trabalharam nas quatro unidades de $\mathrm{CME}$ durante os períodos de 02 a 18 de junho de 2014 e de 04 a 12 de setembro de 2014, de segunda a sexta-feira, das 7:00 às 16:00 horas, sendo quatro (7\%) enfermeiras e 51 (93\%) técnicos/auxiliares/atendentes de enfermagem.

Durante o período de coleta de dados, foram coletadas 1315 amostras do tempo de trabalho executado pelos profissionais de enfermagem nas 15 posições de trabalho das unidades de CME participantes do estudo.

O tempo padrão de execução dos materiais $(T P)_{i}$ em cada posição de trabalho foi a média dos tempos de execução dos kits referentes às quatro unidades de $\mathrm{CME}$, acrescido do fator correspondente ao ritmo do trabalhador (FR) na execução das atividades da sua posição de trabalho, e do valor atribuído à porcentagem do tempo destinado para as tolerâncias correspondentes às necessidades pessoais e de fadiga do trabalhador (FT).

Ao determinar o tempo padrão de processamento de kits $(T P)_{i}$ e identificar a média diária de kits produzidos $\left(\overline{\Re_{i}}\right)$ em cada posição de trabalho, foi possível calcular a carga média diária de trabalho de cada posição de trabalho e a carga média diária total de trabalho $\overline{\mathrm{T}}_{\text {cme }}$ de cada CME estudado.

A síntese dos cálculos realizados está demonstrada na Tabela 1.

Tabela 1 - Cargas médias diárias de trabalho de cada posição de trabalho nas unidades de CME - São Paulo, SP, Brasil, 2014.

\begin{tabular}{ccccccc}
\hline Área & Posições de trabalho & Descrição da atividade & CME & Média diária de kits & Tempo padrão (min) & Carga média diária (horas/dia) \\
\hline & & I & 765 & 1,94 & 24,7 \\
A & \multirow{2}{*}{1} & Recepção e recolhimento & II & 1083 & 1,94 & 35,0 \\
& dos materiais & III & 359 & 1,94 & 11,6 \\
& & IV & 686 & 1,94 & 22,2 \\
\hline
\end{tabular}




\section{...continuação}

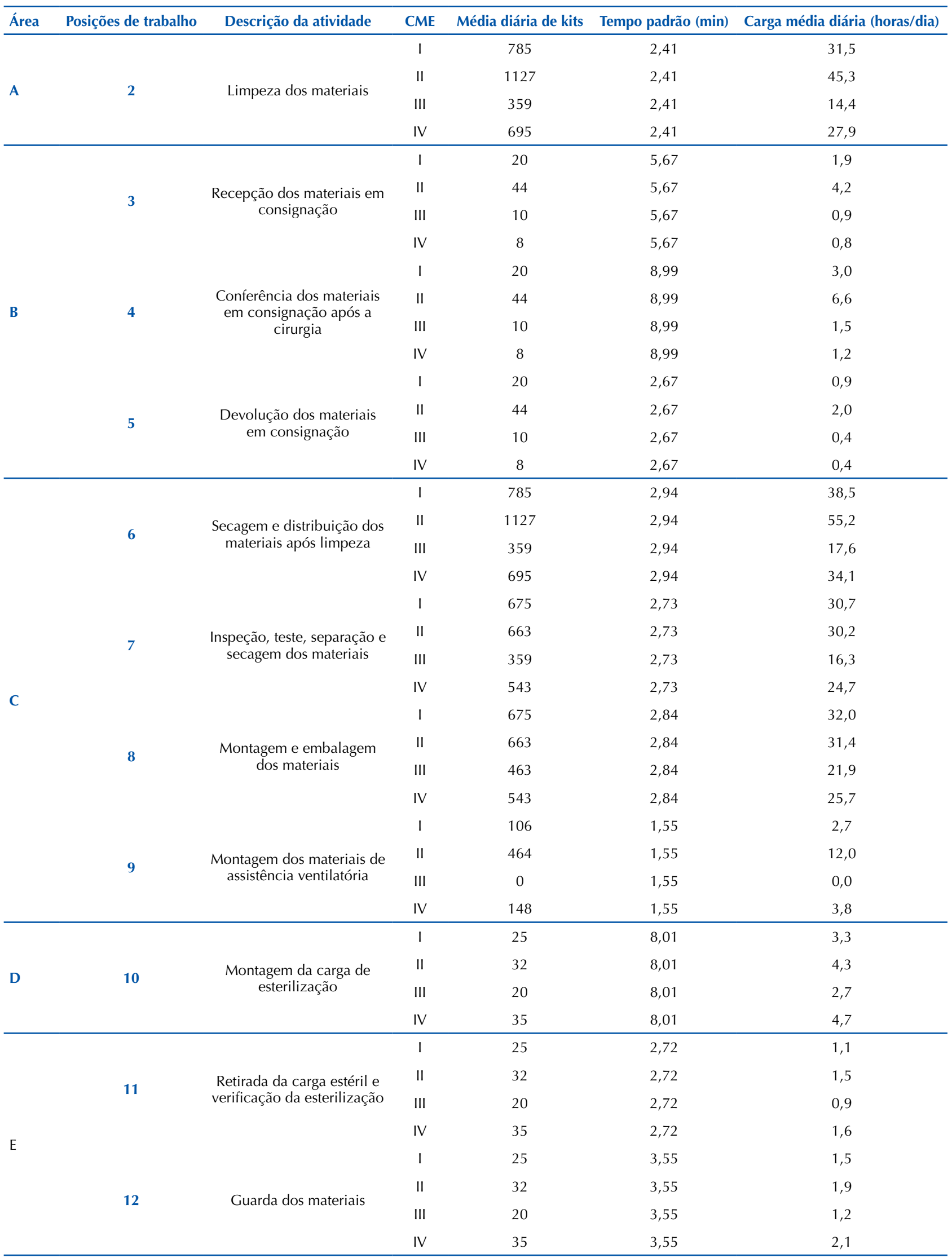

continua... 
...continuação

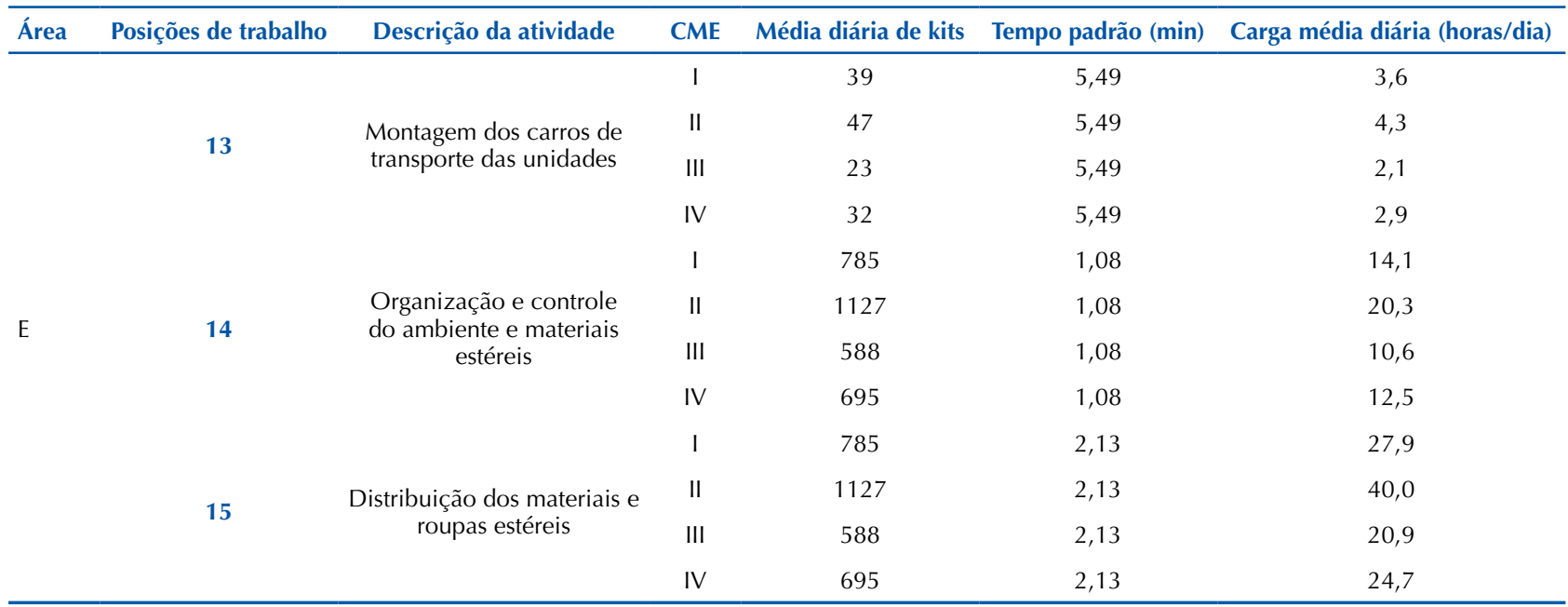

As cargas médias diárias de trabalho das unidades de médias de todas as posições de trabalho, conforme apresenCME foram calculadas pela soma das cargas de trabalho tado na Tabela 2.

Tabela 2 - Cargas médias totais de trabalho de cada CME - São Paulo, SP, Brasil, 2014.

\begin{tabular}{|c|c|c|c|c|c|c|}
\hline \multirow[b]{2}{*}{ Área } & \multirow[b]{2}{*}{ Posições de trabalho } & \multirow[b]{2}{*}{ Descrição da atividade } & \multicolumn{4}{|c|}{ Carga média diária (horas/dia) } \\
\hline & & & CME I & CME II & CME III & CME \\
\hline \multirow{2}{*}{ A } & 1 & Recepção e recolhimento dos materiais & 24,7 & 35,0 & 11,6 & 22,2 \\
\hline & 2 & Limpeza dos materiais & 31,5 & 45,3 & 14,4 & 27,9 \\
\hline \multirow{3}{*}{ B } & 3 & Recepção dos materiais em consignação & 1,9 & 4,2 & 0,9 & 0,8 \\
\hline & 4 & $\begin{array}{c}\text { Conferência dos materiais em consignação } \\
\text { após a cirurgia }\end{array}$ & 3,0 & 6,6 & 1,5 & 1,2 \\
\hline & 5 & Devolução dos materiais em consignação & 0,9 & 2,0 & 0,4 & 0,4 \\
\hline \multirow{4}{*}{ C } & 6 & $\begin{array}{l}\text { Secagem e distribuição dos materiais após } \\
\text { limpeza }\end{array}$ & 38,5 & 55,2 & 17,6 & 34,1 \\
\hline & 7 & $\begin{array}{c}\text { Inspeção, teste, separação e secagem dos } \\
\text { materiais }\end{array}$ & 30,7 & 30,2 & 16,3 & 24,7 \\
\hline & 8 & Montagem e embalagem dos materiais & 32,0 & 31,4 & 21,9 & 25,7 \\
\hline & 9 & $\begin{array}{c}\text { Montagem dos materiais de assistência } \\
\text { ventilatória }\end{array}$ & 2,7 & 12,0 & 0,0 & 3,8 \\
\hline D & 10 & Montagem da carga de esterilização & 3,3 & 4,3 & 2,7 & 4,7 \\
\hline \multirow{5}{*}{$\mathbf{E}$} & 11 & $\begin{array}{c}\text { Retirada da carga estéril e verificação da } \\
\text { esterilização }\end{array}$ & 1,1 & 1,5 & 0,9 & 1,6 \\
\hline & 12 & Guarda dos materiais & 1,5 & 1,9 & 1,2 & 2,1 \\
\hline & 13 & $\begin{array}{c}\text { Montagem dos carros de transporte das } \\
\text { unidades }\end{array}$ & 3,6 & 4,3 & 2,1 & 2,9 \\
\hline & 14 & $\begin{array}{l}\text { Organização e controle do ambiente e } \\
\text { materiais estéreis }\end{array}$ & 14,1 & 20,3 & 10,6 & 12,5 \\
\hline & 15 & Distribuição dos materiais e roupas estéreis & 27,9 & 40,0 & 20,9 & 24,7 \\
\hline \multicolumn{3}{|c|}{ Carga média diária total de trabalho } & 217,4 & 294,0 & 123,1 & 189,1 \\
\hline
\end{tabular}

$\mathrm{Na}$ Tabela 3, é demonstrado o quantitativo de profissionais (técnicos e auxiliares de enfermagem) esperado para cada CME, calculado a partir da carga média diária das unidades, da jornada de trabalho determinada pelas instituições (seis horas) e do índice de segurança técnica (IST) de no mínimo $15 \%$ do total, dos quais $8,3 \%$ referentes a férias e $6,7 \%$ a ausências não previstas (IST $=15 \%$, conforme preconizado pela Resolução COFEN n 543 de $\left.2017^{(4)}\right)$. 
No CME III, foi considerado um IST de $20 \%$ na determinação do quadro de pessoal para a cobertura dos recessos relativos às emendas dos feriados. Como a instituição a que pertence o
CME III tem vínculo direto com uma universidade pública, ela segue o calendário acadêmico elevando o número de dias de folgas referentes a feriados que não coincidem com o domingo.

Tabela 3 - Demonstrativo do quantitativo de pessoal de enfermagem calculado para as unidades de CME - São Paulo, SP, Brasil, 2014.

\begin{tabular}{|c|c|c|c|c|}
\hline \multirow{2}{*}{ VARIÁVEIS } & \multicolumn{4}{|c|}{ CME } \\
\hline & $\mathbf{I}$ & II & III & IV \\
\hline Carga média diária total de trabalho $-\overline{T_{\text {tec }}}$ & 217,4 & 294,0 & 123,1 & 189,1 \\
\hline Jornada de trabalho (horas) - $t$ & 6 & 6 & 6 & 6 \\
\hline Quantidade de profissionais de enfermagem - $q_{\text {tec }}$ & 36 & 49 & 21 & 32 \\
\hline Índice de segurança técnica (IST) & 1,15 & 1,15 & 1,20 & 1,15 \\
\hline Quadro de profissionais de enfermagem esperado $-Q_{c m e}$ & 42 & 56 & 25 & 36 \\
\hline Quadro de profissionais de enfermagem observado & 35 & 52 & 28 & 48 \\
\hline Teste Qui Quadrado & 1,07 & 0,33 & 0,47 & 3,81 \\
\hline Qui Quadrado calculado & \multicolumn{4}{|c|}{5,68} \\
\hline Valor crítico a=0,05 $3 \mathrm{GL}$ & \multicolumn{4}{|c|}{7,82} \\
\hline
\end{tabular}

\section{DISCUSSÃO}

Como a metodologia proposta nesta pesquisa é inédita na área de CME, os resultados apresentados podem ser pouco confrontados com outros estudos.

Várias pesquisas têm sido desenvolvidas em diversas áreas de assistência à saúde explorando o tema carga de trabalho da equipe de enfermagem. Entretanto, durante a revisão da literatura, foi encontrado apenas um estudo ${ }^{(15)}$ com o objetivo de identificar a carga de trabalho da equipe de enfermagem em CME, em que foi empregado um método bastante diferente do da atual investigação, dificultando a comparação dos dados obtidos.

Esse estudo foi desenvolvido com base no elenco de atividades descrito e validado na literatura ${ }^{(2)}$, que favoreceu a determinação da carga de trabalho de profissionais de enfermagem na área de CME.

Os critérios elaborados para selecionar as unidades de CME foram: a centralização das atividades pertinentes ao processamento dos produtos para saúde; utilização de equipamentos nas diferentes etapas do processamento dos materiais; realização de controles de produção nas diferentes áreas da unidade; e a presença de enfermeiras. Tais critérios permitiram escolher locais com bons processos de trabalho, nos quais as atividades realizadas pela equipe de enfermagem estavam em conformidade com os regulamentos e manuais técnicos da área e dessa forma, contempladas no quadro de atividades utilizado no estudo ${ }^{(2)}$.

Não foi objetivo desta pesquisa conhecer o tempo padrão ou estabelecer a carga de trabalho relacionada às atividades próprias dos enfermeiros nas unidades de CME. Entretanto, durante a coleta de dados no CME IV, foi observada a atuação de enfermeiros desempenhando as atividades descritas nas posições de trabalho da Área B - Controle de materiais em consignação. Essas amostras foram consideradas na determinação do tempo padrão, pois interferiam na carga de trabalho e não eram atividades específicas dos enfermeiros nessas unidades.

Em um estudo, foram analisadas as atividades realizadas pelos enfermeiros de CME de instituições hospitalares com base nas atividades específicas dos enfermeiros validadas no estudo utilizado nessa pesquisa ${ }^{(2,16)}$. O resultado desse trabalho demonstrou que 15 atividades são realizadas diariamente e nove mensalmente. As atividades associadas à supervisão e controle do recebimento, uso dos materiais em consignação, e cobrança dos materiais em consignação representaram uma atribuição diária dos enfermeiros, com frequências de realização de $67,75 \%$, $48,39 \%$ e $54,84 \%$, respectivamente. Contudo, em algumas instituições, essas atividades não fazem parte das atribuições das enfermeiras ${ }^{(16)}$.

Para obter o valor médio de tempo de cada uma das 15 posições de trabalho, 1315 amostras de ciclos de trabalho foram mensuradas.

$\mathrm{Na}$ análise do tempo médio selecionado ou tempo médio de processamento de kits nas posições de trabalho, como não houve diferenças significativas entre os valores encontrados nos CMEs, foi obtido o tempo padrão nas diferentes posições de trabalho. Na prática, houve variações de tempo relacionada com a ação dos profissionais ocupando uma mesma posição de trabalho nas amostras e diversificação na quantidade de itens que compunham os conjuntos de materiais, pois as amostras foram colhidas aleatoriamente.

Nesse sentido, é possível afirmar que os processos de trabalho pertinentes ao processamento de artigos médico-hospitalares nas unidades participantes do estudo são semelhantes.

Com relação ao tempo padrão de processamento de materiais, o primeiro e o terceiro maiores valores foram obtidos na Posição de trabalho 4 e na Posição de trabalho 3, onde materiais complexos utilizados nas cirurgias de ortopedia, 
neurocirurgia e cirurgia vídeo assistida são recebidos e conferidos pelos profissionais de enfermagem do CME após a utilização nos procedimentos cirúrgicos.

Esse achado corrobora os resultados de outro estudo ${ }^{(15)}$, que também apontou um tempo maior de processamento para os pacotes classificados como complexos, pois as atividades envolvidas em sua manipulação demandavam maior tempo de trabalho dos profissionais de enfermagem.

O segundo maior tempo padrão foi verificado na Posição de Trabalho 10. Esse resultado foi atribuído ao procedimento de colocar os pacotes para esterilização nos equipamentos, a identificação e descrição da carga e ao uso de instrumentos de controle de rastreabilidade.

A maior carga de trabalho da equipe de enfermagem foi encontrada no CME II (294,0 horas). A produção de kits observada nesta unidade interferiu diretamente neste resultado. Ao analisar a carga de trabalho nas posições de trabalho, em praticamente todas as posições, a carga de trabalho foi maior no CME II, demonstrando que a produção, ou seja, a quantidade de produtos para a saúde processados pelas unidades de CME, interfere diretamente na carga de trabalho destas unidades.

Considerando a carga de trabalho da equipe de enfermagem do CME II, há necessidade maior de pessoal, o que na prática já está estabelecido. $\mathrm{O}$ quadro de profissionais da unidade conta com 52 técnicos de enfermagem, um quantitativo muito próximo do esperado, que corresponde a 56 trabalhadores.

A segunda maior carga de trabalho foi verificada no CME I (217,4 horas). A unidade conta com uma equipe de técnicos de enfermagem (35) inferior ao esperado (42), sugerindo possível sobrecarga de trabalho.

Ao analisar o quadro de pessoal de enfermagem obtido pela aplicação do método proposto nesta pesquisa, com o quadro de pessoal existente no CME I e CME II, os resultados encontrados podem estar associados ao estabelecimento de jornadas extras aos trabalhadores pelas instituições para suprir a necessidade de pessoal. Este fato merece uma investigação em separado, pois justificaria a produção de materiais com um quadro de pessoal menor que o esperado.

No CME IV, a carga de trabalho encontrada foi de 189,1 horas, revelando a necessidade de 36 profissionais de enfermagem. Entretanto, o quadro de técnicos de enfermagem na unidade é de 44 profissionais. $\mathrm{Na}$ obtenção do tempo padrão de processamento dos kits nas posições de trabalho da área de Controle de materiais em consignação, os tempos de execução das atividades realizadas pelos enfermeiros foram considerados na determinação da carga de trabalho da unidade. Dessa forma, esses profissionais foram contabilizados no quadro de pessoal observado (48). Considerando esse raciocínio, o quadro de técnicos observado neste CME é muito superior ao esperado.

A análise deste resultado sugere que a instituição pode não estar funcionando com sua capacidade plena ou necessita rever os processos de trabalho desenvolvidos nas diferentes áreas do CME IV, com o objetivo de esclarecer a real necessidade do quadro observado. Também cabe investigar se há outras variáveis ou atividades desempenhadas pelos profissionais fora da unidade que não foram contempladas no estudo.

Analisando a carga de trabalho nas Posições de trabalho 10,11 e 12 , foram encontrados valores um pouco maiores no CME IV. Esse resultado está associado ao número de equipamentos de esterilização, em maior número nessa unidade, e ao fato de os kits de assistência ventilatória serem esterilizados na autoclave de formaldeído. Estes aspectos aumentam a produção dessas posições de trabalho, quando comparado com o CME I e o CME II, onde esses materiais são submetidos apenas à desinfecção térmica.

A menor carga de trabalho foi verificada no CME III (123,1 horas). Durante a coleta dos dados, a instituição passou por um período de greve dos trabalhadores, fato que pode ter influenciado o resultado obtido, já que a carga de trabalho tem uma relação direta com a produção nas unidades de $\mathrm{CME}$. A redução do número de procedimentos cirúrgicos, ocasionada pela falta de pessoal, pode ter comprometido a produção diária do CME e impactado na determinação da carga de trabalho. Deve-se considerar ainda que as atividades referentes à Posição de Trabalho 9 não são realizadas na unidade, o que influencia diretamente o valor obtido, principalmente em comparação com a carga desta posição de trabalho nas demais unidades.

$\mathrm{Na}$ determinação do quantitativo de pessoal do CME III, foi considerado um IST de 1,20, referente à cobertura adicional para os recessos de emendas dos feriados previstos no calendário da universidade onde a unidade está inserida. $\mathrm{O}$ quadro de pessoal apresentou-se muito próximo do esperado, suscitando questionamentos sobre sua adequação na vigência de elevação da carga de trabalho, nas condições normais de funcionamento da instituição.

Com a finalidade de comparar o quadro de pessoal esperado obtido pela aplicação do método de dimensionamento de pessoal e pelos parâmetros propostos nesse estudo, com o quadro de pessoal observado nas unidades de CME participantes do estudo, foi realizado o teste do Qui quadrado (C2) adotando o nível de significância $\mathrm{a}=0,05$. O resultado desse teste mostrou um valor encontrado $(5,68)$ abaixo do valor crítico $(7,82)$, possibilitando afirmar que o valor calculado não difere do valor observado, considerando o nível de significância de 5\% em todas as unidades.

\section{CONCLUSÃO}

A necessidade de superar a problemática envolvendo aspectos quantitativos e qualitativos de recursos humanos de enfermagem em unidades de CME de instituições hospitalares brasileiras motivou a realização deste estudo.

A presente investigação possibilitou propor parâmetros de tempo para determinar a carga de trabalho, e apresenta-se como importante referencial para auxiliar os enfermeiros na elaboração e justificativa de propostas consistentes para o planejamento, alocação e distribuição de profissionais de enfermagem nessas unidades, considerando a especificidade dos processos de trabalho realizados. A partir da carga de trabalho obtida em cada CME estudado, foi 
possível determinar o quadro de pessoal necessário nessas unidades, avaliar o quantitativo de profissionais existente, e compreender como se distribui o tempo de trabalho dos profissionais de enfermagem nas diferentes posições de trabalho propostas nessa pesquisa.
Recomenda-se utilizar os parâmetros propostos nesta pesquisa em outros cenários de trabalho e que os resultados possam ser confrontados com os apresentados neste estudo, com o objetivo de validar esta metodologia em diferentes unidades de CME do país.

\section{RESUMO}

Objetivo: Determinar a carga de trabalho em Centro de Material e Esterilização como parâmetro para o método de dimensionamento dos profissionais de enfermagem. Método: Estudo de natureza quantitativa, observacional, realizado em quatro instituições hospitalares da cidade de São Paulo, em três etapas: mapeamento das atividades em posições de trabalho; determinação do tempo padrão de processamento dos materiais e estabelecimento da carga média diária de trabalho. Resultados: Foram obtidas 1315 amostras de tempo nas 15 posições de trabalho mapeadas. Os valores de tempo não apresentaram diferenças significativas, possibilitando determinar o tempo padrão de processamento dos materiais. As cargas médias diárias de trabalho corresponderam à: 217,4; 294,0; 123,1 e 189,1 horas. Conclusão: Além de propor um método para determinar a carga de trabalho, a presente investigação indica parâmetros para o processamento de materiais e constitui importante referencial para elaborar propostas consistentes para o planejamento, alocação e distribuição de profissionais de enfermagem nessas unidades.

\section{DESCRITORES}

Recursos Humanos de Enfermagem; Carga de Trabalho; Esterilização; Administração de Materiais no Hospital.

\section{RESUMEN}

Objetivo: Determinar la carga de trabajo en el Centro de Material y Esterilización como parámetro para el método de dimensionamiento del personal de enfermería. Método: Estudio cuantitativo, observacional realizado en cuatro hospitales de la ciudad de São Paulo en tres pasos: mapeo de actividades en puestos de trabajo; determinación del tiempo estándar de procesamiento de materiales; y establecimiento de la carga de trabajo diaria promedio. Resultados: En los 15 puestos de trabajo mapeados, se obtuvieron 1315 muestras de tiempo. Como los valores de tiempo no mostraron diferencias significativas, fue posible determinar el tiempo de procesamiento estándar de los materiales. Las cargas de trabajo diarias promedio correspondieron a 217.4; 294,0; 123,1; y 189,1 horas. Conclusión: Además de proponer un método para determinar la carga de trabajo, el presente estudio indica parámetros para el procesamiento de materiales y constituye una referencia importante para desarrollar propuestas consistentes para la planificación, asignación y distribución de profesionales de enfermería en estas unidades.

\section{DESCRIPTORES}

Personal de Enfermería; Carga de Trabajo; Esterilización; Administración de Materiales de Hospital.

\section{REFERÊNCIAS}

1. Chiavenato I. Gerenciando com as pessoas: transformando o executivo em um excelente gestor de pessoas: um guia para executivo aprender a lidar com sua equipe de trabalho. $9^{\mathrm{a}}$ ed. Rio de Janeiro: Elsevier; 2005.

2. Costa JA, Fugulin FMT. Nursing activities in central supply and sterilization: a contribution to personnel design. Acta Paul Enferm 2011;24(2):249-56.

3. Aiken LH, Sloane DM, Bruyneel L, Heede KV, Griffiths P, Busse R, et al. Nurse staffing and education and hospital mortality in nine European countries: a retrospective observational study. Lancet. 2014;383(9931):1824-30.

4. Conselho Federal de Enfermagem (COFEN). Resolução n. 543/2017. Atualiza e estabelece parâmetros para o Dimensionamento do Quadro de Profissionais de Enfermagem nos serviços/locais em que são realizadas atividades de enfermagem [Internet]. Brasília; 2017 [citado 2019 maio 02]. Disponível em: http://www.portalcofen.gov.br.

5. Polit DF, Beck CT. Fundamentos de pesquisa em enfermagem. $7^{\mathrm{a}}$ ed. Porto Alegre: Artes Médicas; 2011.

6. Graziano KU, Silva A, Psaltikidis EM, organizadoras. Enfermagem em Centro de Material e Esterilização. São Paulo: Manole; 2011.

7. Brasil. Ministério da Saúde; Agência Nacional de Vigilância Sanitária. Resolução RDC n. 15, de 15 de março de 2012. Dispõe sobre requisitos de boas práticas para o processamento de produtos para saúde e dá outras providências [Internet]. Brasília; 2012. [citado 2012 mar. 19] Disponível em: http://www.brasilsus.com.br/legislacoes/gm/112548-15.html

8. São Paulo (Estado). Secretaria de Estado da Saúde; Divisão de Infecção Hospitalar, Centro de Vigilância Epidemiológica. Manual de avaliação da qualidade de práticas de controle de infecção hospitalar [Internet]. São Paulo; 2006. [citado 2007 out. 13]. Disponível em: http://www.cve.com.br.

9. Associação Paulista de Estudos e Controle de Infecção Hospitalar (APECHI). Reprocessamento de artigos de uso único. São Paulo; 2008.

10. Sociedade Brasileira de Enfermagem em Centro Cirúrgico, Recuperação Anestésica e Centro de Material e Esterilização (SOBECC). Práticas recomendadas - SOBECC. $7^{\mathrm{a}}$ ed. São Paulo; 2017.

11. Organização Nacional de Acreditação (ONA). Manual de Acreditação das Organizações Prestadoras de Serviços Hospitalares. Brasília; 2006.

12. Association for the Advancement of Medical Instrumentation. ANSI/AAMI ST79: 2017 Comprehensive guide to steam sterilization assurance in health care facilities. Arlington; 2017.

13. Association of Peri Operative Registered Nurses. Standards, Recommended Practices, and Guidelines. Recommended practices for sterilization. Denver: AORN; 2013. 
14. Barners MR. Estudo de tempos e movimentos: projeto e medida de trabalho. São Paulo: Edgard Blücher; 1977.

15. Neis MEB, Gelbcke FL. Carga de trabalho em centro de material e esterilização. Rev SOBECC. 2014;19(1):11-7.

16. Gil RF, Camelo SH, Laus AM. Atividades do enfermeiro de centro de material em instituições hospitalares. Texto Contexto Enferm [Internet]. 2013 [citado 2015 Jul 09];22(4):927-34. Disponível em: http://www.scielo.br/pdf/tce/v22n4/08.pdf

(cc) BY Este é um artigo em acesso aberto, distribuído sob os termos da Licença Creative Commons. 